\title{
Moxifloxacin resistance in the FI5/LAM4/KZN extensively drug-resistant strain of Mycobacterium tuberculosis
}

\author{
Navisha Dookie \\ A Willem Sturm \\ Prashini Moodley \\ Department of Infection Prevention \\ and Control, Nelson R Mandela \\ School of Medicine, School of \\ Laboratory Medicine and Medical \\ Science, College of Health Science, \\ University of KwaZulu-Natal, \\ Durban, South Africa
}

This article was published in the following Dove Press journal:

Infection and Drug Resistance

19 August 2014

Number of times this article has been viewed
Objectives: Moxifloxacin (MXF) has been advocated for the treatment of extensively drugresistant (XDR) tuberculosis despite resistance to older-generation fluoroquinolones. We investigated the relationship between the minimum inhibitory concentration (MIC) of MXF and mutations in the gyrA and gyrB genes in Mycobacterium tuberculosis (MTB) isolates from KwaZulu-Natal (KZN) Province of South Africa.

Materials and methods: MICs of 56 MTB isolates were compared to the mutations in the quinolone resistance-determining region known to confer fluoroquinolone resistance. Isolates were genotyped by IS6110 restriction fragment length polymorphism analysis.

Results: The circulating F15/LAM4/KZN XDR strain circulating in KZN Province harbored the A90V mutation and displayed high-level resistance with MICs of $8 \mathrm{mg} / \mathrm{L}$ for ciprofloxacin and ofloxacin and $\geq 1 \mathrm{mg} / \mathrm{L}$ for MXF.

Conclusion: The inclusion of MXF in XDR-TB treatment regimens requires careful consideration in our setting, where clinical outcome data in MXF-containing regimens are unavailable.

Keywords: fluoroquinolones, susceptibility testing, strain typing, drug-resistance

\section{Introduction}

The human immunodeficiency virus (HIV) pandemic has fuelled the tuberculosis (TB) epidemic by creating a population of immunosuppressed individuals that are highly susceptible to Mycobacterium tuberculosis (MTB) infection. The last decade has seen an unprecedented increase in antimycobacterial drug resistance. Of the estimated 1.3 million deaths resulting from TB globally in 2012, 13.1\% of these deaths were due to drug resistance. Appropriate treatment of patients with drug-resistant strains of MTB is of vital importance in limiting the transmission of the disease and reducing mortality rates. ${ }^{1}$

Fluoroquinolones are potent antibiotics that have been used in clinical practice since the early 1980s. ${ }^{2}$ They display broad-spectrum antimicrobial activity, and have been used extensively in the treatment of bacterial infections of the respiratory, gastrointestinal, and urinary tracts, as well as in sexually transmitted diseases and chronic osteomyelitis. ${ }^{3}$ The fluoroquinolones have been advocated for the treatment of patients with multidrug-resistant (MDR) MTB, defined as resistance to at least isoniazid and rifampicin. Patients with extensively drug-resistant (XDR) MTB harbor MDR TB strains with additional resistance to fluoroquinolones and one of the second-line antimycobacterial injectable (kanamycin, amikacin, and capreomycin) agents. However, moxifloxacin (MXF), a new-generation fluoroquinolone, has been recommended by 
the World Health Organization (WHO) for the treatment of XDR. Studies that have explored the efficacy of MXF against XDR strains of MTB have concluded that the drug may be used in XDR cases provided that the infecting isolate has a minimum inhibitory concentration (MIC) of $<2 \mathrm{mg} / \mathrm{L}$ for $\mathrm{MXF}^{1,4}$

MXF differs in structure when compared to ofloxacin (OFX) and ciprofloxacin (CPX). The structural difference, which includes a methoxy group in the C-8 position of $\mathrm{MXF}$, results in increased bactericidal activity of the drug, lower MICs, and a lower propensity for the development of resistance to the drug. ${ }^{5}$ Although cross-resistance has been reported, it has been argued that the increased bactericidal activity of MXF and the lower MIC allow for this drug to be effective against XDR isolates where CPX and OFX are ineffective. ${ }^{3,4}$

The fluoroquinolones inactivate the deoxyribonucleic acid (DNA) gyrase enzyme, thereby preventing transcription during cell replication. DNA gyrases are encoded by the $g y r A$ and $g y r B$ genes. Fluoroquinolone-resistant strains of MTB most frequently display mutations on codons 90, 91, and 94 of the gyr $A$ gene. ${ }^{6-9}$ Additionally, double mutations in the $g y r A$ or concomitant $g y r A$ and $g y r B$ mutations have been reported. ${ }^{6,8}$ The level of fluoroquinolone resistance is dependent on the mutation in the resistance-conferring gene and the fluoroquinolone tested. ${ }^{10}$ Studies have demonstrated that MIC levels of resistant isolates are higher for oldergeneration fluoroquinolones than for MXF. ${ }^{6-9}$

The use of MXF in XDR treatment regimens was introduced without prior testing for susceptibility against the circulating XDR isolates in KwaZulu-Natal (KZN) Province. The aim of this study was to correlate the minimum inhibitory concentration (MIC) levels of the fluoroquinolones with mutations in the $g y r A$ and $g y r B$ genes in a subset of clinical isolates from the KZN Province of South Africa.

\section{Materials and methods}

\section{Clinical isolates}

The isolates used in this study were retrieved from the culture collection in the Department of Infection Prevention and Control, Nelson R Mandela School of Medicine, School of Laboratory Medicine and Medical Science, College of Health Science, University of KwaZulu-Natal. We included the following phenotypes: ten fully drug-susceptible (DS), $20 \mathrm{MDR}$, and $30 \mathrm{XDR}$. The isolates were collected between 2005 and 2008 from patients in Umzinyathi District, KZN, South Africa. H37Rv was included as the reference strain. Ethical approval for the study (BREC 247/09) was granted by the Biomedical Research Ethics Committee at the University of KwaZulu-Natal.

\section{MIC determination}

MIC determination of the drugs was performed by means of the agar dilution method using Middlebrook 7H10 (BD, Franklin Lakes, NJ, USA) media supplemented with oleic acid-albumin-dextrose-catalase (BD). The drug concentrations used ranged from 0.03 to $8 \mathrm{mg} / \mathrm{L}$ for $\mathrm{CPX}$, OFX (Sigma-Aldrich, St Louis, MO, USA), and MXF (Bayer, Leverkusen, Germany). Following inoculation, plates were incubated in a $\mathrm{CO}_{2}(5 \%)$-enriched atmosphere at $37^{\circ} \mathrm{C}$ for 21 days. MIC values were recorded as the lowest concentration of the drug that resulted in complete inhibition of growth. The cutoff value for resistance $\geq 2 \mathrm{mg} / \mathrm{L}$ for CPX and OFX according to WHO recommendations ${ }^{11}$ and $\geq 0.5 \mathrm{mg} / \mathrm{L}$ for MXF, as described by Angeby et al. ${ }^{12}$ All MIC experiments were carried out in triplicate.

\section{DNA extraction and PCR}

DNA was extracted using cetyl-trimethyl-ammonium bromide-sodium chloride, as previously described. ${ }^{13}$ The quinolone resistance-determining region (QRDR) and flanking regions of the $g y r A$ and $g y r B$ genes were amplified using primer pairs designed for this study: gyrA forward (CGATTGCAAACGAGGAATAG), gyrA reverse (GGCCAGTTTTGTAGGCATCA), and $g y r B$ forward (ATCAACCTGACCGACGAGAG), gyrB reverse (GCCGAGTCACCTTCTACGAC). ${ }^{14}$ Polymerase chain reaction (PCR) was performed using the Expand highfidelity PCR system (dNTPack; Hoffman-La Roche, Basel, Switzerland).

Cycling conditions were as follows: initial denaturation at $94^{\circ} \mathrm{C}$ for 2 minutes; 40 cycles of denaturation at $94^{\circ} \mathrm{C}$ for 45 seconds, annealing at $53^{\circ} \mathrm{C}($ gyr $A)$ or $56^{\circ} \mathrm{C}($ gyrB) for 45 seconds and extension at $72^{\circ} \mathrm{C}$ for 45 seconds; and a final extension of 7 minutes at $72^{\circ} \mathrm{C}$. The quality of PCR amplicons was checked on a $1 \%$ agarose gel.

Sequencing PCR products were purified using the Invitrogen PureLink ${ }^{\circledR}$ PCR purification kit (Thermo Fisher Scientific, Waltham, MA, USA). The sequencing reactions were performed using an ABI Prism BigDye ${ }^{\circledR}$ Terminator cycle-sequencing kit 3.1 (Thermo Fisher Scientific) with the same forward primers as used for PCR amplification. Geneious version 5.5.7 sequenceanalysis software was used to identify mutations in the final nucleotide sequences in comparison to the MTB H37Rv reference strain. ${ }^{15}$ 


\section{Genotyping}

Genotyping was performed by IS6110 restriction fragment length polymorphism (RFLP) analysis using the Southern blot hybridization method, as previously described. ${ }^{16}$

\section{Results}

Isolates

Of the 60 isolates selected, four MDR isolates did not grow sufficiently on retrieval subculture and were excluded from further analysis. MIC and sequencing data were therefore obtained for ten DS, $16 \mathrm{MDR}$, and $30 \mathrm{XDR}$ isolates.

\section{MIC results}

The DS and MDR isolates displayed MICs for CPX, OFX, and MXF in the susceptible range. All 30 XDR isolates tested displayed MICs for CPX, OFX, and MXF that were in the resistant range (Table 1).

\section{Sequencing of the gyrA and gyrB genes}

Three mutations were observed in the nucleotide sequence of the gyrA gene: E21Q (GAA $\rightarrow$ CAA), S95T (ACG $\rightarrow$ ACC), and $\mathrm{A} 90 \mathrm{~V}(\mathrm{GCG} \rightarrow \mathrm{GTG})$. E21Q and S95T were present in all 56 isolates, regardless of MIC values (Table 1). The A90 V mutation was present only in the $30 \mathrm{XDR}$ isolates, correlating with an MIC value of $8 \mathrm{mg} / \mathrm{L}$ for CPX and OFX. In the case of MXF, 23 of the 30 XDR isolates had an MIC value of $2 \mathrm{mg} / \mathrm{L}$, while seven had an MIC value of $1 \mathrm{mg} / \mathrm{L}$.

Table I RFLP strain families of Mycobacterium tuberculosis isolates stratified by MIC and resistance conferring mutations in the gyrA gene

\begin{tabular}{|c|c|c|c|c|c|}
\hline \multirow[t]{2}{*}{ Strain family } & \multirow{2}{*}{$\begin{array}{l}\text { Isolates, } n \\
\text { (phenotype) }\end{array}$} & \multirow{2}{*}{$\begin{array}{l}\text { Mutations } \\
\text { associated } \\
\text { with } \\
\text { resistance }\end{array}$} & \multicolumn{3}{|c|}{ MIC (mg/L) $)^{a}$} \\
\hline & & & CPX & OFX & MXF \\
\hline \multirow[t]{4}{*}{ FI5/LAM4/KZN } & 23 (XDR) & A90V & 8 & 8 & 2 \\
\hline & 7 (XDR) & A90V & 8 & 8 & I \\
\hline & 4 (MDR) & None & I & I & 0.5 \\
\hline & I (MDR) & None & 1 & I & 0.25 \\
\hline \multirow[t]{4}{*}{ F28 } & 5 (MDR) & None & I & I & 0.5 \\
\hline & 3 (MDR) & None & 1 & 1 & 0.25 \\
\hline & I (MDR) & None & 0.5 & 0.5 & 0.5 \\
\hline & I (MDR) & None & 0.5 & 0.5 & 0.25 \\
\hline LAM3/FII & I (DS) & None & 0.5 & 0.5 & 0.125 \\
\hline Beijing family & 6 (DS) & None & 0.5 & 0.5 & 0.125 \\
\hline \multirow[t]{2}{*}{ Unique } & 3 (DS) & None & 0.5 & 0.5 & 0.125 \\
\hline & I (MDR) & None & 0.5 & 0.5 & 0.25 \\
\hline
\end{tabular}

Note: aResistance defined as $2 \mathrm{mg} / \mathrm{L}$ for CPX and OFX and $0.5 \mathrm{mg} / \mathrm{L}$ for MXF.

Abbreviations: RFLP, restriction fragment length polymorphism; MIC, minimum inhibitory concentration; CPX, ciprofloxacin; OFX, ofloxacin; MXF moxifloxacin; XDR, extensively multidrug-resistant; MDR, multidrug-resistant; DS, drug-susceptible.
Ten MDR isolates with an MIC for MXF at the proposed breakpoint for resistance, ie, $0.5 \mathrm{mg} / \mathrm{L}$, did not display the A90V mutation. No mutations were detected in the $\operatorname{gyr} B$ genes of the isolates tested.

\section{IS6 I I 0 restriction fragment length polymorphism analysis}

IS6110 RFLP analysis revealed that 35 of the 56 isolates belonged to the F15/LAM4/KZN family of strains. This included all 30 XDR isolates and five MDR isolates. Seventeen of the remaining isolates belonged to recognized strain families (F28, F11, and Beijing) while four showed a unique RFLP profile.

\section{Discussion}

We report on the correlation between MICs of MXF and mutations in the gyrA gene in a selection of clinical isolates in KZN, South Africa. Fluoroquinolone resistance in MTB is most frequently attributed to mutations occurring in the QRDR of the gyrA gene. The QRDR of the gyrA gene consists of a short region, coding for amino acids 74-113. In our study, we sequenced the QRDR of both the gyrA and gyrB genes, as well as flanking regions. We found the C269T mutation within the QRDR of the gyrA gene, which corresponds with the amino acid change $\mathrm{A} 90 \mathrm{~V}$, correlated with the high MICs seen in the XDR MTB isolates that we studied. The A90V mutation in gyrA has been described as one of the most frequent mutations associated with fluoroquinolone resistance. ${ }^{17}$ In our study, based on WHO-recommended breakpoints, the $\mathrm{A} 90 \mathrm{~V}$ mutation in XDR isolates was linked to resistance in all three fluoroquinolones tested. ${ }^{11}$

We did not find mutations in $\operatorname{gyr} B$ in any of the isolates tested. This supports previous observations that mutations within the $\operatorname{gyr} B$ gene are rare in MTB. ${ }^{8,17}$ Maruri et al conducted a systematic review to evaluate gyrase mutations associated with fluoroquinolone resistance in MTB. The study reported on 534 fluoroquinolone-resistant MTB isolates, of which 17 (3\%) harbored mutations within the QRDR of the gyrB gene. In addition, four different numbering systems were used to report on mutations in the $\operatorname{gyr} B$ gene, resulting in major discrepancies. The authors proposed a uniform numbering system in an attempt to improve the molecular detection in the gyrases. ${ }^{18}$ The significance of mutations within the QRDR of the gyrB gene cannot be ignored, and is thought to play an important role in resistance. ${ }^{9}$

WHO guidelines propose $0.5 \mathrm{mg} / \mathrm{L}$ as the breakpoint MIC for susceptibility testing of MXF in the BACTEC ${ }^{\text {TM }}$ 460 system (BD) and $0.25 \mathrm{mg} / \mathrm{L}$ in the BACTEC MGIT 
960 (BD) system. ${ }^{11}$ Breakpoints for other test systems are not proposed. Angeby et al demonstrated comparable MIC results for MXF on Middlebook 7H10 agar and the BACTEC 460 system. ${ }^{12}$ With Middlebrook 7 H10 plates used for MIC determination, all our XDR isolates had MICs of $>0.5 \mathrm{mg} / \mathrm{L}$. As per the WHO definition, these are classified as MXF-resistant, and this implies that MXF should not be recommended for treatment of cases harboring such isolates. However, there are reports of MXF efficacy in isolates with MXF MICs $<2 \mathrm{mg} /$ L. 3,4,6,10,19

Poissy et al used the murine model to demonstrate that MXF is effective against OFX-resistant strains. They reported that MXF was most effective on MTB strains with MICs $\geq 0.5 \mathrm{mg} / \mathrm{L}$. Reduced mortality was observed in mice infected with strains, with MICs $\geq 2 \mathrm{mg} / \mathrm{L}$ compared to untreated controls. ${ }^{4}$ Fillion et al demonstrated similar findings using the murine model to determine the effect of a multidrug regimen containing $\mathrm{MXF}$. The sterilizing activity of the multidrug regimen decreased in strains with increased MICs to MXF. The impact of the sterilizing activity of the most effective second-line treatment regimen (ie, ethionamide, pyrazinamide, amikacin, and MXF) is dependent on the MIC of MXF, and thus the MIC of MXF has to be determined for all strains resistant to OFX. Mice infected with strains with MXF MICs of $0.5 \mathrm{mg} / \mathrm{L}$ and $4 \mathrm{mg} / \mathrm{L}$ recorded relapse rates of $50 \%$ and $86 \%$, respectively, compared to the wild type. ${ }^{10}$

Sirgel et al proposed that MXF and OFX are possibly not equally affected by mutations associated with fluoroquinolone resistance. ${ }^{6}$ They concluded that the use of MXF for the treatment of infection with OFX-resistant strains is justified when combined with other drugs. They further suggested that the low recommended breakpoint of $0.5 \mathrm{mg} / \mathrm{L}$ determining MXF resistance may therefore give a false impression of clinical inactivity. Poissy et al and Sirgel et al support WHO recommendations on the use of MXF for the treatment of XDR provided that the infecting isolate has an MXF MIC of $<2 \mathrm{mg} / \mathrm{L}$. 4,6

Feasey et al reported on a case where high-dose MXF (600 mg/day) in combination with PZA, CAP, LIN, PAS, and amoxicillin clavulanic acid for 22 months successfully treated a case with an infecting isolate that was resistant to isoniazid, rifampicin, ethambutol, prothionamide, OFX, streptomycin, and MXF (MIC of $2 \mathrm{mg} / \mathrm{L}$ ). While high-dose MXF treatment increases the peak plasma (MXF), resulting in levels that remain constantly above the MIC, it is difficult to assess the exact role of MXF in the successful management of this patient, since other drugs with known efficacy were also included in the treatment regimen. ${ }^{19}$
Jacobson et al conducted a meta-analysis to assess treatment outcomes in patients with XDR. The report summarized 13 studies conducted mainly amongst HIV-uninfected people, who received a new-generation fluoroquinolone together with other anti-TB drugs. They concluded that the addition of MXF to XDR regimens may improve outcomes, but further evaluation in clinical trials is warranted. ${ }^{3}$

In $\mathrm{KZN}$, the regimen used for XDR treatment is a combination of drugs with proven and putative efficacy. The National Department of Health in South Africa recommends the use of MXF as part of XDR treatment in the presence of OFX resistance. Fluoroquinolones are added despite in vitro reports of resistance in the hope that there may be some residual activity. It is debatable whether the perceived benefit of using MXF under these circumstances outweighs the risks caused by side effects of this drug or the increased exertion of antibiotic pressure in the era of ever-increasing drug resistance. ${ }^{20}$ Mendel and Springsklee ${ }^{21}$ warned that the use of newer-generation fluoroquinolones in patients who display low-level resistance will be disastrous from a public health perspective. The use of MXF in such cases will result in the ready emergence of highly resistant strains unless drug concentrations are sustained above mutant-prevention concentrations at all infection sites. The latter is extremely difficult to achieve, and thus the use of MXF in patients with resistance to older-generation fluoroquinolones will only further drive resistance among XDR strains of MTB.

Although all isolates used in this study were from different patients, the XDR isolates displayed a high degree of similarity and belonged to a single genotype, ie, F15/LAM4/ KZN. To date, all reports from KZN have attributed XDR to this strain. Ramtahal showed that the spread of XDR in KZN was clonal with the F15/LAM4/KZN strain. ${ }^{22}$ Clonal spread of this strain has been ongoing since at least $2005 .{ }^{23}$ During this period, further acquisition of resistance may have occurred. We therefore performed susceptibility testing and sequencing on $30 \mathrm{XDR}$ isolates belonging to the only XDR strain family currently in KZN. Seven of the isolates had MICs of $1 \mathrm{mg} / \mathrm{L}$, and 23 had MICs of $2 \mathrm{mg} / \mathrm{L}$. Basic microbiological principles regarding in vitro determination of MICs allows for one twofold MIC variation between tests. This implies that our XDR isolates may in their most susceptible form have MICs between 0.5 and $1 \mathrm{mg} / \mathrm{L}$ and in their most resistant form from 2 to $4 \mathrm{mg} / \mathrm{L} .{ }^{24}$ Gandhi et al found that a large variety of strains were associated with DS, and this decreased as the degree of resistance increased. The low diversity of strains driving the MDR and XDR epidemics supports the theory of clonal expansion of drug-resistant 
phenotypes in KZN. This picture is different in other parts of South Africa. In the Eastern and Western Cape Provinces, the Beijing strain is accountable for the majority of XDR. Strains responsible for MDR and XDR in other provinces include the S, T1, and other families. The reasons for geographic differences remain uncertain. ${ }^{25}$

\section{Conclusion}

Regardless of the strain family implicated in infection with $\mathrm{XDR}$, the breakpoint for resistance to MXF remains the subject of debate. Our results support concerns regarding the use of MXF in KZN. While there may be a role for MXF as part of individualized XDR treatment regimens, this cannot be advocated as part of empiric treatment protocols in the absence of MXF MIC data of the circulating XDR strains in an area. In addition, validation from larger population-based studies using MXF in combination with various other antidrug regimes must be conducted. Early bactericidal assays with MXF will also give useful data to inform our practice.

\section{Disclosure}

The authors report no conflicts of interest in this work.

\section{References}

1. World Health Organization. Global Tuberculosis Report 2013. Geneva: WHO; 2013. Available from: http://www.who.int/iris/bitstream/10665/ 91355/1/9789241564656_eng.pdf?ua=1. Accessed July 9, 2014.

2. Ginsburg AS, Grosset JH, Bishai WR. Fluoroquinolones, tuberculosis, and resistance. Lancet Infect Dis. 2003;3(7):432-442.

3. Jacobson KR, Tierney DB, Jeon CY, Mitnick CD, Murray MB. Treatment outcomes among patients with extensively drug-resistant tuberculosis: systematic review and meta-analysis. Clin Infect Dis. 2010;51(1):6-14.

4. Poissy J, Aubry A, Fernandez C, et al. Should moxifloxacin be used for the treatment of extensively drug-resistant tuberculosis? An answer from a murine model. Antimicrob Agents Chemother. 2010;54(11): 4765-4771.

5. Dalhoff A. Comparative in vitro and in vivo activity of the C-8 methoxy quinolone moxifloxacin and the C-8 chlorine quinolone BAY y 3118 . Clin Infect Dis. 2001;32 Suppl 1:S16-S22.

6. Sirgel FA, Warren RM, Streicher EM, Victor TC, van Helden PD, Böttger EC. gyrA mutations and phenotypic susceptibility levels to ofloxacin and moxifloxacin in clinical isolates of Mycobacterium tuberculosis. J Antimicrob Chemother. 2010;67(5):1088-1093.

7. Wang JY, Lee LN, Lai HC, et al. Fluoroquinolone resistance in Mycobacterium tuberculosis isolates: associated genetic mutations and relation to antimicrobial exposure. J Antimicrob Chemother. 2007;59(5):860-865.

8. Von Groll A, Martin A, Jureen P, et al. Fluoroquinolone resistance in Mycobacterium tuberculosis and mutations in gyrA and gyrB. Antimicrob Agents Chemother. 2009;53(10):4498-4500.
9. Malik S, Willby M, Sikes D, Tsodikov OV, Posey JE. New Insights into fluoroquinolone resistance in Mycobacterium tuberculosis: functional genetic analysis of gyrA and gyrB mutations. PLoS One. 2012;7(6): 39754

10. Fillion A, Aubry A, Brossier F, Chauffour A, Jarlier V, Veziris N. Impact of fluoroquinolone resistance on bactericidal and sterilizing activity of a moxifloxacin-containing regimen in murine tuberculosis. Antimicrob Agents Chemother. 2013;57(9):4496-4500.

11. World Health Organization. Guidelines for Surveillance of Drug Resistance in Tuberculosis. 4th ed. Geneva: WHO; 2009. Available from: http://www.who.int/entity/tb/publications/2009/surveillance_ guidelines/en/index.html. Accessed February 21, 2013.

12. Angeby KA, Jureen P, Giske CG, et al. Wild-type distributions of four fluoroquinolones active against Mycobacterium tuberculosis in relation to current critical concentrations and available pharmacokinetic and pharmacodynamic data. J Antimicrob Chemother. 2010;65(5):946-952.

13. van Soolingen D, Hermans PW, de Haas PE, Soll DR, van Embden JD. Occurrence and stability of insertion sequences in Mycobacterium tuberculosis complex strains: evaluation of an insertion sequencedependent DNA polymorphism as a tool in the epidemiology of tuberculosis. J Clin Microbiol. 1991;29(11):2578-2586.

14. Rozen S, Skaletsky HJ. Primer3: Pick primers from a DNA sequence. 2009. Available from: http://frodo.wi.mit.edu/primer3. Accessed January 14, 2014.

15. Geneious [website on the Internet]. Available from: http://www. geneious.com. Accessed January 14, 2014.

16. Van Embden JD, Cave MD, Crawford JT, et al. Strain Identification of Mycobacterium tuberculosis by DNA fingerprinting: recommendations for a standardized methodology. J Clin Microbiol. 1993;31(2):406-409.

17. Da Silva PE, Palomino JC. Molecular basis and mechanisms of drug resistance in Mycobacterium tuberculosis: classical and new drugs. J Antimicrob Chemother. 2011;66(7):1417-1430.

18. Maruri F, Sterling TR, Kaiga AW, et al. A systematic review of gyrase mutations associated with fluoroquinolone-resistant Mycobacterium tuberculosis and a proposed gyrase numbering system. J Antimicrob Chemother. 2012;67(4):819-831.

19. Feasey NA, Pond M, Coleman D, et al. Moxifloxacin and pyrazinamide susceptibility testing in a complex case of multidrug-resistant tuberculosis. Int J Tuberc Lung Dis. 2010;15(3):417-420.

20. South Africa Department of Health. Management of Drug-Resistant Tuberculosis: Policy Guidelines. Pretoria: Department of Health; 2011.

21. Mendel C, Springsklee M. Moxifloxacin for tuberculosis. Lancet Infect Dis. 2012;12(3):176-177; author reply 177-178.

22. Ramtahal MA. Spread of Multidrug Resistant Tuberculosis (MDR) Including Extensively Drug Resistant Tuberculosis (XDR), in Rural KwaZulu-Natal, South Africa [master's thesis]. Durban: University of KwaZulu-Natal; 2012.

23. Gandhi NR, Moll A, Sturm AW, et al. Extensively drug-resistant tuberculosis as a cause of death in patients co-infected with tuberculosis and HIV in a rural area of South Africa. Lancet. 2006;368(9574): $1575-1580$.

24. Clinical and Laboratory Standards Institute. Methods for Dilution Antimicrobial Susceptibility Tests for Bacteria That Grow Aerobically: Approved Standard. 9th ed. Wayne (PA): CLSI; 2012. Available from: http://antimicrobianos.com.ar/ATB/wp-content/uploads/2012/11/03CLSI-M07-A9-2012.pdf. Accessed May 14, 2014.

25. Gandhi NR, Brust JCM, Moodley P, et al. Minimal diversity of drug-resistant Mycobacterium tuberculosis strains, South Africa. Emerg Infect Dis. 2014;20(3):426-433. 
Infection and Drug Resistance

Dovepress

\section{Publish your work in this journal}

Infection and Drug Resistance is an international, peer-reviewed openaccess journal that focuses on the optimal treatment of infection (bacterial, fungal and viral) and the development and institution of preventive strategies to minimize the development and spread of resistance. The journal is specifically concerned with the epidemiology of antibiotic

resistance and the mechanisms of resistance development and diffusion in both hospitals and the community. The manuscript management system is completely online and includes a very quick and fair peerreview system, which is all easy to use. Visit http://www.dovepress.com/ testimonials.php to read real quotes from published authors.

Submit your manuscript here: http://www.dovepress.com/infection-and-drug-resistance-journal 